\title{
Comparative abilities of different herbivorous species to graze in upland areas: consequences for productivity and vegetation
}

\author{
JA Milne \\ Macaulay Land Use Research Institute, Craigiebuckler, Aberdeen AB9 2QJ, UK
}

Animal size, as it influences digestive function and ingestive behaviour, is the most important factor influencing animal production and vegetation change. These attributes of size, digestive function and ingestion capability allow ruminants to be classified generally as grazers, browsers or mixed grazers/browsers. Within each class there remains, however, considerable variation in ingestive behaviour. Results of experiments, in which sheep and cattle grazed semi-natural vegetation, show that grazing time and selectivity are greater for sheep than cattle. This supports the general theory that maintaining diet quality is more important for smaller ruminants. Bite mass per unit of liveweight is also greater for sheep than cattle and, taken with the higher energy requirements of cattle, suggests that sheep are more adapted to grazing semi-natural vegetation than cattle. In heterogeneous vegetation, such as that found in upland areas, there is considerable opportunity for choice of diet. Evidence suggests that the digestibility and the short-term rate of intake of plant species by sheep and cattle and the biomass of these plant species are important determinants of which plant species are selected by these animal species from heterogeneous pastures found in upland areas. There are, however, differences between cattle and sheep in the manner in which they graze such pastures and an example is given of the complementarity that can be achieved by grazing both species together, not only in increasing the productivity of sheep but also in increasing the biological diversity of the pasture. The complexity of the process of grazing heterogeneous upland pastures makes it necessary to develop computer models to predict, for example, the consequences of management changes. Such a model has been developed and used to achieve the above objective in relation to sheep grazing the upland areas of the UK. 